\title{
Graphische Rechentafeln (Nomogramme) für die Berechnung der ganzen rationalen Funktion.
}

\author{
Yon Alexayogr Fischen (in Prag).
}

Uebersicht. - Es wird, wnter Hevanziehung der einfachsten Hilfsmittel der Nomographie, die Bildung der ganzen rationalen Euntion sowohl in reellen als auch im komplexen Bereich auf nomographisch-graphischem Weg gezeigt.

In den bekannteren Lehrbüchern der praktischen Analysis werden zur zeichnerischen Bildung der ganzen rationalen Funktion die Verfahren von I. A. v. Segner und E. LILL. allenfalls die Verallgemeinerung und Aus* dehnung des letzteren auf des komplexe Gebiet durch C. Bunge behandelt. In einer vor einiger Zeit erschienenen Arbeit gibt ferner H. Behmann (1) (*) eine Vereinfachung des v. SEgrerschen Verfahrens. Ich möchte nun im folgenden eine wohl noch einfachere, auf nomographischer Grundlage beruhende Behandlungsweise der ganzen rationalen Funktion

$$
y_{n}=a_{0} x^{n}+a_{1} x^{n-1}+\ldots+a_{n-1} x+a_{n}
$$

mitteilen, die den Vorzug hat, auch auf die komplexe Funktion

$$
w_{n}=a_{0} z^{n}+\alpha_{1} z^{n-1}+\cdots+a_{n-1} z+a_{n}
$$

(mit komplexem Argument und komplexen Koeffizienten) ausgedehnt werden zu können. Sie geht von der, sowohl dem v. SEGNER - bzw. BeHManN -, als auch dem LmLschen Verfahren gemeinsamen Zerlegung der Gleichung $\left(I^{\prime}\right)$ in die Gleichungskette

$$
y_{v+1}=y_{v} x+a_{v+1}
$$

$\left(v=0,1, \ldots, n-1, y_{0} \equiv a_{0}\right)$ aus.

a) Reelles Gebiet: Hier wird die einfache Gestalt von (II') benutzt, um durch abwechselnde Anwendung der übereinandergelagerten Tafeln für die beiden, schon in jedem einführenden Lehrbuch der Nomographie ausführlich

(*) Die Zahlen in Klammern beziehen sich auf den Schriftennachweis am Ende der Arbeit. 
behandelten, einfachsten "kanonischen Formen»

$$
\begin{aligned}
& f_{1}\left(x_{1}\right)+f_{2}\left(x_{2}\right)=f_{3}\left(x_{3}\right) \\
& f_{1}\left(x_{1}\right)=f_{2}\left(x_{2}\right) f_{3}\left(x_{3}\right)
\end{aligned}
$$

die Gleichung (I') in Form eines einzigen ununterbrochenen Linienzuges zu bilden. Abb. 1 zeigt die zu beachtende Vorzeichonwahl bei der Verquickung

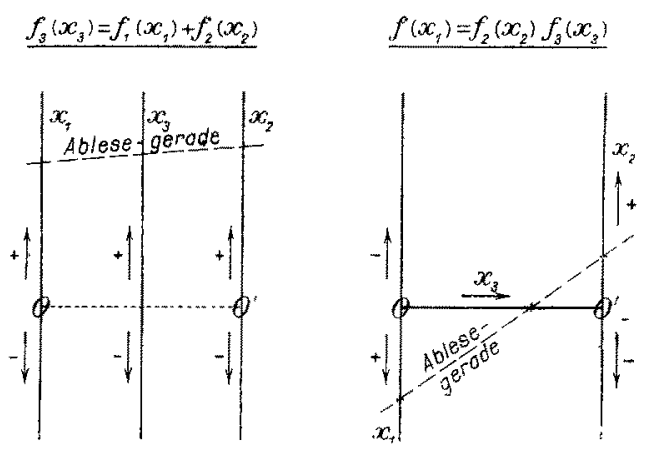

Abb. 1

beider Formen. Die Abb. 2, die das Bersprex

$$
y_{2}=x^{2}+2 x-3
$$

für $x=2$ zeigt, dürfte durch Angabe der Schritte wohl sofort verstandlich werden.

SoHRITT 1: Bildung von $a_{n}\left(\equiv y_{0}\right)$ auf der rechten Leiter.

SohritT 2: Bildung von $-a_{0} x$ auf der linken Leiter (Verwendung von (b)). SchritT 3: Bildung von $y_{\text {: }}$ auf der rechten Leiter gemäss $-a_{0} x+y_{1}=a_{1}$ (Verwendung von (a)).

SoHrimT 4: Bildung von - $y_{1} x$ auf der linken Leiter (Verwendung von (b)).

SchrIn' 5: Bildung von $y_{2}$ auf der rechten Leiter gemäss $-y_{1} x+y_{2}=a_{2}$ (Verwendung von (a)).

Die linke Leiter dient also bloss als «Zapfenlinie», trägt daher keine Leiter. Führt man das in Abb. 2 dargestellte Nomogramm auf durohsichtigem Papier oder Zellhorn aus und bringt für verschiedene Werte von $x$ die entsprechenden $y$-Werte über die zugehörigen $x$-Werte (Millimeterpapier als Grundblatt!), so kann die so entstehende verzerrte $y$-Kurve ohneweiteres z. B. zur zeichnerischen Lösung der Gleichung $y_{n} \equiv 0$ verwendet werden.

ß) Komplexes Gebiet: Da die Bildung von (I') aus (II') rein formaler Natur ist, gilt sie insbesondere auch für komplexes Argument und ebensolche 
Koeffizienten. Es sei

$$
\left.\begin{array}{rl}
w_{\nu+1} & =u_{\nu+1}+i v_{\nu+1} \\
z & =\xi+i \eta \\
a_{\nu+1} & =\alpha_{\nu+1}+i \beta_{\nu+1}
\end{array}\right\} \vee=0,1, \ldots n-1, u_{0} \equiv \alpha_{0},
$$
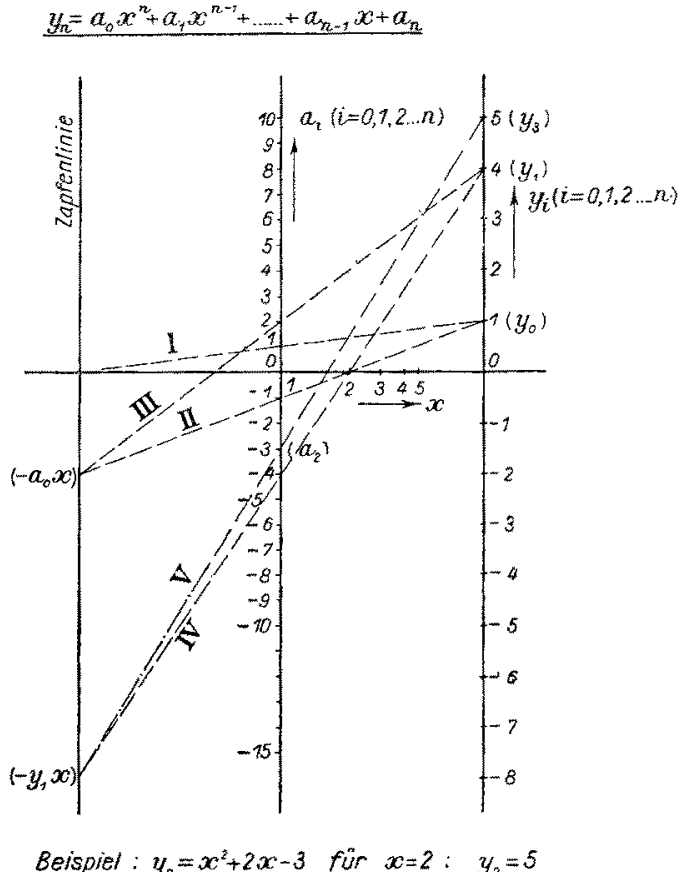

Abb. 2

Dann ergibt die zu (II') analoge Gleichungskette

$\left(\mathrm{II}^{\prime \prime}\right)$

$$
w_{\nu+1}=u_{\nu+1}+i v_{\nu+1}=\left(u_{\nu}+i v_{\nu}\right)(\xi+i \eta)+\alpha_{\nu+1}+i \beta_{\nu+1}
$$

das Gleichungskettenpaar

$$
\begin{aligned}
& u_{\nu+1}=u_{\nu} \xi-v_{y} \eta+\alpha_{\nu+1} \\
& v_{\nu+1}=v_{\nu} \xi+u_{\nu} \eta+\beta_{\nu+1}
\end{aligned}
$$

Dies ist behufs nomographischer Verwertung folgendermassen zu schreiben:

$$
\begin{aligned}
& -u_{y} \xi+u_{y+1}=\zeta_{1}=-v_{y} \eta+\alpha_{\nu+1}, \\
& -v_{y} \xi+v_{y+1}=\zeta_{z}=u_{v} \eta+\beta_{y+1} .
\end{aligned}
$$

Hierin sind $\zeta_{1}$ und $\zeta_{2}$ Zapfenwerte auf den bezüglichen Zapfenlinien. 
Bei der Bildung von $u_{y+1}$ ist so vorzugehen: (s. Abb. 3a)

Schrime 1: Bildung von $-v, \eta$ auf der linken Leiter (Verwendung von (b)).

Schritr 2: Bildung von $\zeta_{1}$ gemäss: $\zeta_{1}=-v_{\nu} \eta+\alpha_{y+1}$ auf der Zapfenlinie $\zeta_{1}$ (Verwendung von (a)).

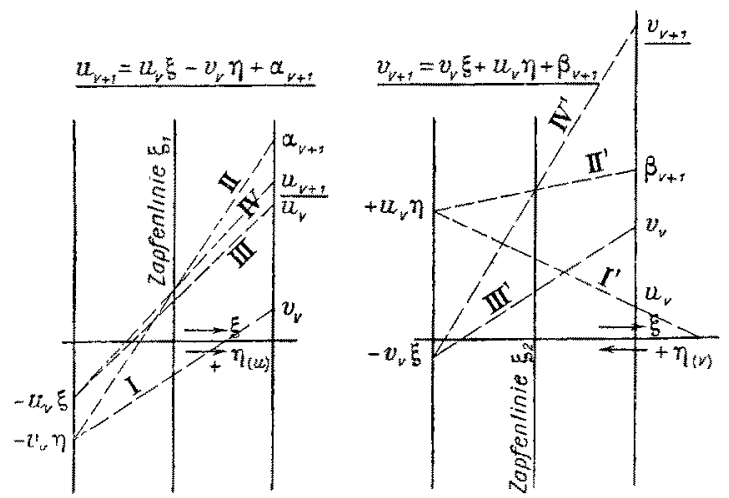

Abb. 3

SoHRIme 3: Bildung von - $u, \xi$ anf der linken Leiter (Verwendung von (b)).

SoHRITT 4: Bildung von $\boldsymbol{u}_{y+1}$ unter Benutzung von $\zeta_{1}$ auf der rechten Leiter (Verwendung von (a)).

Ganz analog ist $v_{v+1}$ zu bilden ( $\mathrm{Abb}$. 3b). Hierbei ist jedoch die zu benutzende $\eta$-Leiter zu «verziffern », d. h., um die Grösse $u_{\nu} \eta$ sinnentsprechend zu bilden, sind positive und negative Werte der fritheren $\eta$-Leiter zu vertauschen. $\mathrm{Zu}$ diesem Behufe sind die beiden $\eta$-Leitern mit den Zeigern $(\mathrm{a})$ bzw. (v), also $\eta_{(u)}$ und $\eta_{(v)}$ versehen worden.

Wie leicht ersichtlich, kann die Bildung von $u_{y_{f-1}}$ and $v_{v_{+1}}$, also schliesslich von $w_{n}$, in einer Tafel erfolgen, da die $\xi$ und $\eta_{(x)}$-Leitern zusammenfallen, daher auf dem einen Ufer der waagrechten Mittelleiter Platz finden, während deren zweites die $\eta_{(v)}$-Leiter trägt. Wie ersichtlich, ist anch hier die linke Leiter bloss Zapfenlinie.

Abb. 4 zeigt das Beispiel:

$$
w_{1}=(2+i) z+3+2 i
$$

fitr $z=1+2 i$. Es ergibt sich: $w_{1}=3-t-7 i$.

Anmerkungen: 1) Der Gedanke, die Nomographie mit dem gewönlichen "Graphischen Rechnen» zu koppeln, ist im vorliegenden Fall nicht neu. Vgl. hierzu (5), S. 254 und 286 (Verfahren von FarId Boulad). Wie ich in einigen Arbeiten (2), (3), (4) zeigen konnte, ist diese auf nomographischer 
Grundlage beruhende Weiterbildung des gewöhnlichen «Graphischen Rechnens », die ich mit dem Namen «Nomographisch-graphisches Rechnen» belegt habe und für die sich übrigens insbesondere in den technischen Wissenschaften verschiedene Sonderfälle vorfinden, eine sehr wesentliche Erwei-

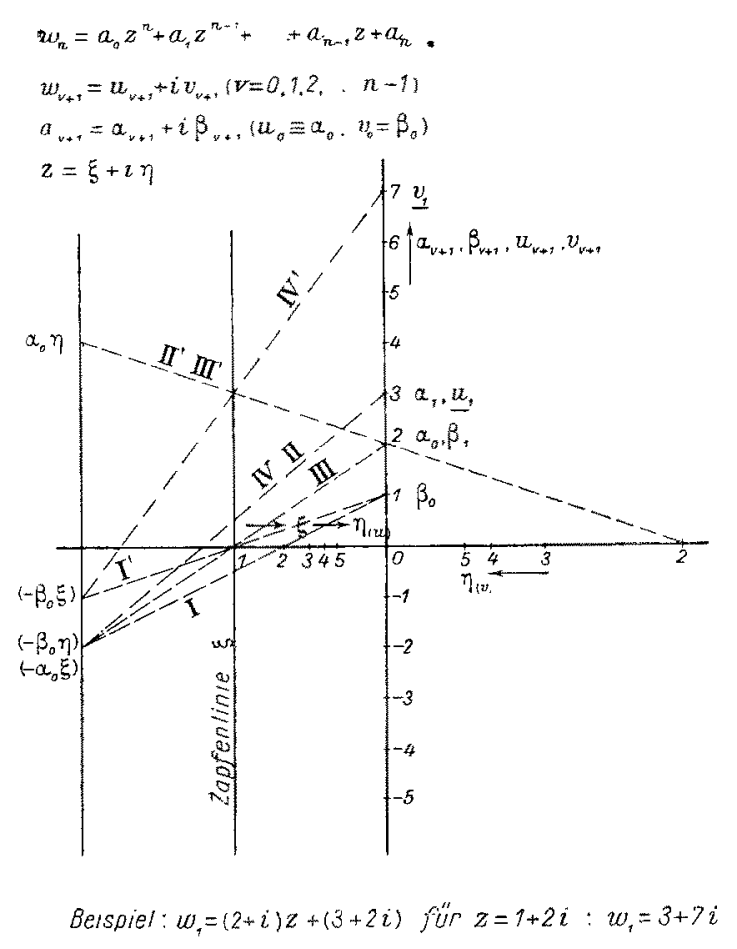

Abb. 4

terung der Nomographie. Es ist daher in diesem Sinne die von M. D'OCaGne (6) rorgeschlagene Einteilung der geometrischen und mechanischen Rechenver* fahren durch einen, zwischen Punkt 4 (Calcul nomographique) und 5 (Calcul nomomécanique) einzuschaltenden weiteren Punkt *Calcul nomographographique $» z u$ vervollständigen.

2) Wie nur kurz erwähnt sei, kann das Vorstehende ohne wesentliche Schwierigkeiten auch für kompliziertere derartige Falle, ferner z. B. zur Summenbildung unendlicher Reihen auf nomographisch-graphischem Wege herangezogen werden, worauf aber nicht weiter eingegangen sei. 


\section{SOHRIFTTUM}

1 - H. BenmaNN, Zur graphischen Behandlung der ganzen rationalen Funhtion. "Z. f. ange* wandte Math. u. Mechanik», 11 (1931), H. 6, S. 463.

2 - A. Fischer, Ueber eine Anwendung des nomographiseh-graphischen Rechnens auf eine Aufgabe aus der technischen Schwingungslehre. "HDT-Mitteihngen des Hauptvereines dentscher Ingenieure in der Tschechoslowakischen Rep. ", 1982, H. 14.

3 - A. Fiscrer, Graphische Ermittung der Scheinteistungsdanerlinie. (Zuschrift zur gleichnamigen Arbeit von H. Kunze). * Elektrotechnik a. Maschinenbau », 1982, H. 45.

4 - A. FiscHer, Deber das allgeneine "Integralrelief» zur nomographisch-graphischen Lösung von Randwertaufgaben genöhnhicher linearer Differentialgleichungen 2. Ondunngdas reelle Gegenstück zum "Sinus-und Tangensrelief in der Etektrotechnik ", von Fritz Emde, "HDI-Mitt. », 1938, H. 1/2.

5 - M. D'OCAGNh, Calcul graphique et Nomographie, 3e ed., Paris, 1924.

6 - M. D'Odagne, Le Calcul simplifié par les procédés mécaniques et graphiques, 30 éd., Paris. 1928. 